\title{
Mortalidad Evitable. El Caso de la Frontera Norte de México, 1980-1990
}

\author{
Avoidable Mortality. The Case of the North border of Mexico, 1980-1990
}

\author{
Carlos Antonio G. Molina' \\ Maria Victoria L. López
}

\section{MOLINA, C. A. G. \& LOPEZ, M. V. L. Avoidable Mortality. The Case of the North border of Mexico, 1980-1990. Cad. Saúde Públ., Rio de Janeiro, 11 (3): 395-407, Jul/Sep, 1995.}

When the death causes are understood as a result of morbility process that may have been avoided with diferent kinds of economic, social and welfare services policies and the advance of scientific and technologic knowledges, there is clearly a structural determinism so that many population social sectors can't reach the «modern epidemiologycal patterns». This study used annual data bases of register death 1979-1991 bringed by INEGI/ DGE-SSA from five Mexican federal border entities. It were adjusted by Preston-Coale method and Life Tables were generated in 1980 and 1990. The percentage contributions of avoidable causes' groups by sex and age of the increases in the Expectation of Life (Ex) were calculated in the same years following Pollard's method.

As result of this research, limited increments of Ex were finded because of the permanence of high contribution of deatlhs, that may have been avoided (50\%). Contrary to popular beliefs, Ex male diminished the difference compared with Ex female. Highest male mortality is explained even for this kind of causes, where accidents and violence deaths are bringing high percentages of total mortality.

Key words: Mortality; Avoidable Mortality; Avoidable Death Causes

\section{INTRODUCCION}

No cabe duda acerca del importante descenso de la mortalidad general e infantil ocurrida en el país y de que la estructura de la mortalidad por causas ha experimentado cambios. Sin embargo, se conoce poco acerca de cómo se da este proceso regionalmente, cómo afecta la estructura de la mortalidad por causas y cómo ellas definen perfiles de mortalidad específicos.

Se parte de considerar que los cinco estados fronterizos: Baja California, Coahuila,

\footnotetext{
${ }^{\prime}$ Centro Regional de Investigaciones Multidisciplinarias (CRIM-UNAM) Apartado Postal 4-106. C.P. 64431. Cuernavaca. Morelos. México

${ }^{2}$ Escuela de Salud Pública de México (ESPM-INSP). Av. Universidad 655, Col Sta María Ahuacatitlán. Cuernavaca, Morelos. México.
}

Chihuahua, Sonora y Tamaulipas presentan, a su interior y en conjunto, formas de enfermar y de morir específicas a sus particulares procesos socio-económicos. Se pretende construir los perfiles de mortalidad con base en sus estructuras de mortalidad por causas y su evolución en el tiempo. Se excluye para este estudio al Estado de Nuevo León ya que tiene pocos movimientos trans-fronterizos aunque económicamente es uno de los más importantes del Norte del país.

La Clasificación Internacional de Enfermedades (CIE) (OMS, 1978) permite, además de considerar los daños a la salud según su localización anatómica y su fisiopatología para lo que fue concebida, ahondar en la comprensión de la distribución y determinantes histórico-sociales de los procesos mórbidos. Se trata de hacer acercamientos a otras realidades diferentes a aquellas para lo que fue creada. 
Para avanzar en lo anterior se planteó retomar dicha clasificación y cualificarla reconstruyendo el dato de mortalidad por causas. Esta reconstrucción se refiere a organizar la información buscando que el dato se transforme en hecho epidemiológico, mediante la articulación de esta información con el análisis del contexto-histórico social determinado (López. 1991). Para ello se rescatan conceptos como los de evitabilidad (Taucher, 1978, 1980: García et al., 1994) que permiten un reacrrupamiento de las causas de muerte factibles de concebir como partes de un proceso, que trasciende lo biológico y lo individual señalando su articulación y delimitación con las condiciones históricosociales, lo cual permite identificar problemas y plantear propuestas de transformación.

Este reagrupamiento define las enfermedades en Evitables y Difícilmente

Evitables según condiciones socioeconómicas, características de prestación de los servicios de salud, calidad del diagnóstico médico y finalmente de los avances del conocimiento científico y técnico en salud. En oposición al exagerado uso y abuso de técnicas estadisticas «modernas», interesa explorar aquí el uso de técnicas demográficas aplicadas a información estadística secundaria y cómo ellas permiten analizar procesos tan complejos como los de salud-enfermedad y muerte. Para estudiar este proceso usando técnicas desarrolladas por disciplinas «formales» como la Demografía o la Epidemiología, se requiere no solamente reconceptualizar sus objetos de estudio sino también aprovechar sus arsenal teóricometodológico con una visión multidisciplinaria.

\section{CONSIDERACIONESTEORICO- METODOLOGICAS}

\section{Consideraciones Generales}

\section{La Frontera Norte de México como Contexto}

Aunque difícil de definir, se entiende aquí la Frontera Norte como un espacio socioeconómico inserto en un proceso de internacionalización estructural (Negrece, 1990).
Entre las principales características sociodemográficas de esta región se destacan la influencia del proceso migratorio y un rápido crecimiento poblacional que supera al registrado en el resto de la República Mexicana y sólo comparable con el observado en las principales zonas metropolitanas en los períodos de mayor expansión (Corona, 1991).

Actualmente, las Entidades fronterizas ocupan, por su extensión territorial, la tercera parte del territorio nacional; sin embargo, la densidad poblacional es baja (14 habitantes por kilómetro cuadrado) lo cual representa el $12.5 \%$ de la población del país.

Durante los últimos 30 años la población de la frontera norte aumentó casi tres veces, y aunque la tasa bruta de natalidad y la tasa bruta de mortalidad registraron un descenso importante en este período, las tasas de crecimiento, total de los estados superaron el promedio nacional, con excepción de la década 1970-1980. El proceso migratorio, aunque con diferencias en los distintos Estados, se caracteriza, de manera general, por un decremento de la inmigración interna e incremento de la emigración interna e internacional (Corona, 1991).

Se trata de Estados con mayor proporción de población urbana, ubicada en la zona mas cercana a la frontera, cuya fuerza de trabajo está principalmente ocupada en actividades industriales, entre las cuales destaca la industria maquiladora para la exportación, actividades comerciales y de servicios, con creciente participación femenina y una importante proporción de fuerza de trabajo transmigrante que trabaja en Estados Unidos aunque su residencia habitual está en México (Cruz, 1992).

\section{Fuentes de Información y Análisis de Datos}

\section{Fuente de Datos}

La información de mortalidad corresponde a las bases de datos anuales de defunciones 1979-1991, generadas por la Secretaría de Salud (SSA) e por el Instituto Nacional de Geografía e Informática (INEGI). Con base en los objetivos del estudio, se seleccionaron las 
variables: Causa básica de muerte, desagregada a tres dígitos, según la Novena Clasificación Internacional de Enfermedades (CIE), Municipio y el tamaño de la localidad de residencia habitual del difunto, sexo, edad y certificación médica.

\section{Calidad y Tratamiento de la Información}

El análisis de las frecuencias de todas las variables en sus códigos unitarios para los años 1979 a 1991 no mostró anormalidades de ninguna especie. Se observó una perfecta correspondencia entre las causas de defunción y la Clasificación Internacional de Enfermedades a tres dígitos (Novena revisión). Los porcentajes de no certificación médica, de subregistro y de causas mal definidas están por debajo de los promedios encontrados para México y de los sugeridos como tolerables para hacer estudios de mortalidad por causas (Jaspers \& Orellana, 1991; Rodriguez, 1989). Lo anterior sugiere que los archivos anuales de defunciones ofrecen información confiable al menos en lo que concierne a las variables que se usan en el estudio.

Para eliminar fluctuaciones anuales que se deben mas al sistema de registro que a cambios reales de la mortalidad, se construyó una serie de defunciones anuales suavizada a través de promedios móviles trienales. Esto quiere decir que un año cualquiera se obtuvo a partir del promedio aritmético de él y los años adyacentes. Este procedimiento produjo una serie ajustada de registros anuales de defunción entre 1980 y 1990.

Para corregir el subregistro de las defunciones se utilizaron técnicas indirectas que usan información de Censos y encuestas, además de los registros de defunción. Las defunciones de mayores de cinco años por sexo y edad fueron evaluadas y ajustadas a través de una técnica que parte de los supuestos de: comportamiento estable de la población, cerrada a la migración y declaración correcta de la edad (Preston \& Coale, 1984). El procedimiento consiste en establecer una relación entre el número de defunciones en un año dado y el número de personas en la población. Aunque los supuestos del procedimiento no se cumplan cabalmente, es posible estimar porcentajes mínimos de subregistro teniendo en cuenta el efecto de la migración, que en el caso de la Frontera Norte es significativa (ONU, 1986; Bennett \& Horiuchi, 1984).

Es importante anotar que, con estos procedimientos, no es posible obtener estimaciones precisas del subregistro, dada la calidad de las defunciones en cuanto a la mala declaración de la edad y al no cumplimiento cabal de los supuestos. Sin embargo, es posible evaluar el posible efecto de ese subregistro y hacer correcciones que estén por debajo de los porcentajes reales. De esta manera, se pretende respetar la información original y rescatar de ella lo que aportan sus diferenciales, no mediendo con precisión sus niveles.

La mortalidad infantil y juvenil se estimó a partir de información de los censos de 1980 y 1990 sobre hijos tenidos nacidos vivos e hijos sobrevivientes de las mujeres en edad fértil clasificadas en grupos quinquenales de edad. La idea fundamental del procedimiento consiste en establecer una relación entre la proporción de hijos tenidos nacidos vivos que han fallecido según la edad de la madre y la probabilidad de morir entre el nacimiento y una edad determinada, que también depende de la edad materna.

Aunque los supuestos de fecundidad constante en un período reciente y de buena declaración de la edad, entre otros, no se cumplen cabalmente, aquí se utiliza una variante a la técnica original de W. Brass (1974), propuesta por J. Trussel (ONU, 1986) que, entre otras ventajas, tiene en cuenta cambios recientes de la mortalidad y refiere las estimaciones $n$ años antes del censo. En general, esta técnica ha permitido hacer estimaciones relativamente confiables ya que es poco sensible al no cumplimiento de sus supuestos (García, 1986; Gómez de León \& Partida, 1993).

A pesar de la evaluación y el suavizamiento realizado a los datos, no se puede desconocer las limitaciones que tienen estas fuentes de información en términos de mala declaración, registro inadecuado y sesgos administrativos que afectan el sistema estadístico en su conjunto. Por otro lado, las estimaciones 
indirectas no son más que eso:

estimaciones que tratan de corregir algunos problemas de información, pero que no pueden ser considerados como mediciones de los niveles de mortalidad, al menos en este trabajo. Los resultados que aquí se presentan deben ser interpretados teniendo en cuenta este hecho.

\section{Variables e Indicadores Utilizados}

\section{Estructura de Mortalidad}

Para el estudio de la mortalidad por causas, se consideró la distribución por sexo y grupos quinquenales de edad para cada uno de los agrupamientos según evitabilidad. se analizaron según su distribución porcentual, número de orden y aporte porcentual y en años de la ganancia en el período de la esperanza de vida al nacimiento. Esta distribución se interpretó como la estructura de la mortalidad por causas, permitiendo hacer comparaciones entre subgrupos poblacionales.

\section{Perfil de Mortalidad}

Se construyeron a partir de las estructuras de mortalidad definidas anteriormente. Se consideró que estas estructuras asumen comportamientos específicos de acuerdo a los distintos niveles de determinación considerados. Se entiende como perfil de mortalidad de un espacio socio-económico específico (Frontera Norte) a las características de los riesgos de muerte y que son observables a través de la estructura de mortalidad por causas, género y grupos de edad, que lo hacen diferenciable de otros espacios y que son la concreción de procesos de salud-enfermedad-muerte históricamente determinados. El perfil, más que un listado de principales causas de muerte, es una síntesis histórica del proceso salud-enfermedad-muerte. Que expresa, entre otras cosas, las contribuciones a la mortalidad de las condiciones de vida, la política social del estado y la prestación de servicios de salud.

\section{Tablas Abreviadas de Mortalidad para 1980 y 1990}

Una vez ajustadas las defunciones, se calcularon las tasas de mortalidad por sexo y grupos quinquenales de edad para el total de las cinco entidades federativas, insumo básico para la construcción de las tablas de mortalidad. Las funciones de la tablas de mortalidad construidas fueron:

- Las probabilidades de morir entre las edades exactas $\mathrm{x}, \mathrm{x}+\mathrm{n}$ años;

- Los sobrevivientes a la edad exacta $\mathrm{x}$ años;

- Defunciones entre las edades exactas $\mathrm{x}$, $\mathrm{x}+\mathrm{n}$ años;

- Población estacionaria (años vividos) entre las edades exactas $\mathrm{x}, \mathrm{x}+\mathrm{n}$ años;

- Años por vivir de los sobrevivientes a la edad exacta x años y;

- La esperanza de vida a la edad exacta x años.

Estas funciones son calculadas para cada sexo, siendo la esperanza de vida al nacimiento el número promedio de años que se espera viva un recien nacido si llegara a experimentar las condiciones de mortalidad estimadas para su población. Los procedimientos seguidos en la construcción de las tablas de mortalidad fueron los recomendados de acuerdo a los tipos de datos disponibles (Ortega, 1987).

\section{Contribución de los Grupos de Causas Evita- bles al Cambio en la Esperanza de Vida y al Diferencial por Sexo. Procedimiento de Pollard}

El razonamiento del procedimiento parte de la afirmación de que una reducción de intensidad en la fuerza de la mortalidad (función que expresa la mortalidad en una edad exacta), en un intervalo pequeño de edades, produce necesariamente un incremento en la esperanza de vida al nacer de la población. Es decir, cada reducción de la mortalidad en una edad individual puede ser calculada teniendo en cuenta el efecto principal y los distintos efectos de interacción sobre la estructura de la mortalidad (Pollard, 1988, 1990;

Rodriguez, 1989). Para ello es necesario 
disponer de las funciones de la tabla de mortalidad, como son los sobrevivientes a una edad exacta y las probabilidades de sobrevivencia en dos momentos en el tiempo. Para medir el efecto de un conjunto de causas, se pondera las probabilidades de muerte de cada grupo de edad por el peso porcentual de ese grupo de causas; normalmente este tipo de estimaciones se hace para cada sexo.

Al igual que los procedimientos anteriores, este no garantiza el cabal cumplimiento de los supuestos, sobre todo aquel que tiene que ver con las interacciones. Pues es posible que una muerte que pudo ser evitada tenga múltiples opciones de serlo y presentar interacción incluso con las dificilmente evitables. Por otro lado, no se reproducen exactamente las diferencias por sexo, lo que puede ser atribuible a los procedimientos de ponderación $\mathrm{y}$ al hecho de que las relaciones exactas solo se lograrían si se consideran las variables como continuas y no discretas como se hace para efectos prácticos. En todo caso es posible corregir este tipo de errores (Pollard, 1990).

\section{Los Agrupamientos de Causas de Muerte como Variables Asociadas a las Condiciones SocioEconómicas y de Servicios de Salud}

Una forma de medir las necesidades en salud es analizar cuáles causas de muerte están afectando mayoritariamente la mortalidad de la población y relacionar esta situación con la manera como la política social y en particular la política en salud presenta una respuesta social organizada. Igualmente, es posible asociar las condiciones de salud con la forma como se distribuyen socialmente los beneficios del desarrollo en términos de infraestructura social y condiciones de vida de la población.

Se definen 9 grupos:

1. Evitables por vacuna o tratamiento preventivo.

2. Evitables por diagnóstico y tratamiento médico precoz.

3. Evitables por condiciones de saneamiento ambiental.
Evitables por acciones mixtas o un conjunto de medidas en política social y de salud como son:

4. Infecciones de las vías respiratorias;

5. Perinatales y de la primera infancia;

6. Complicaciones del embarazo, parto y puerperio, cirrosis hepática y tuberculosis;

7. Accidentes, envenenamientos y violencias;

8. Difícilmente evitables por el estado actual del conocimiento científico y desarrollo tecnológico;

9. Mal definidas y otras causas no clasificables en los grupos anteriores.

Al grupo de evitables por vacuna o tratamiento preventivo, corresponden patologías asociadas con algunas medidas preventivas, como la inmuno-prevención y el establecimiento de acciones de vigilancia, educación para la salud, control y regulación de ambiente, etc. Algunas de las patologías de este grupo son: difteria, tosferina, fiebre reumática. En el grupo de evitables por diagnóstico y tratamiento médico, se incluyen los padecimientos que dependen de las características cualitativas y cuantitativas de prestación de servicios de salud, expresadas en patologías como: úlcera, gastritis y algunos tumores. Al grupo de evitables por condiciones de saneamiento ambiental corresponden aquellos padecimientos cuya condición de evitabilidad está dada, fundamentalmente, por la mejoría en las condiciones del medio ambiente a través del control, de la prevención y de la erradicación de ciertos factores de riesgo y que son expresión de las condiciones materiales de existencia de la población. En este grupo se incluyen patologías infecciosas como: fiebre tifoidea, cólera y salmonelosis.

$\mathrm{Al}$ grupo de evitables por acciones mixtas corresponden los padecimientos evitables con un conjunto de medidas que se asocian a la prestación de los servicios médicos y a acciones de orden económico social más amplio. Se considera que estos grupos están directamente asociados con las condiciones del desarrollo y en él se incluyen patologías 
como: las infecciones agudas de las vías respiratorias superiores, los accidentes, envenenamientos y violencias, las complicaciones del embarazo, parto y puerperio.

En el grupo de los padecimientos difícilmente evitables, se consideran aquellas patologías de difícil manejo en el estado actual del conocimiento y desarrollo tecnológico en salud como son: leucemia, esclerósis múltiple, diabetes mellitus y linfosarcoma, entre otras.

\section{Procesamiento de Datos y Análisis Estadístico}

Los datos fueron procesados en SPSS/PC+, los promedios móviles trienales fueron procesados electrónicamente y cada distribución numérica y/o porcentual se refiere al promedio móvil mientras no se diga lo contrario. El análisis estadístico es descriptivo y bivariado.

\section{RESULTADOS}

\section{Diferenciales de Mortalidad por Causas Según Evitabilidad}

Como se puede observar en la Gráfica 1, las causas de muerte evitables representan alrededor del $50 \%$ del total de muertes en toda la Frontera y el descenso en los 13 años es mínimo. Su descenso anual fue del 1.2\%.

En las causas mal definidas se puede ver el porcentaje que aportan como un criterio de evaluación de los datos. Este está por debajo del 5\% y descendió de manera importante en el período. El grupo de "otras" podría decirse que ha permanecido estable en la década.

Cuando se compara la estructura porcentual de las causas de muerte según condiciones de evitabilidad en los cinco estados, se perciben pocas diferencias. Al igual que lo observado para el total de la frontera, es mínimo el descenso en el porcentaje de causas evitables. Para 1990 Coahuila presenta el menor porcentaje de muertes evitables (46.2); Baja California, Chihuahua y Sonora presentan, todavía, un $50 \%$ de defunciones que pudieron haber sido evitadas. Aunque pequeñas, estas diferencias dan cuenta de distintas realidades de saludenfermedad-muerte en los distintos estados fronterizos.

Considerando el aporte de los distintos subgrupos de muertes evitables con respecto al total de defunciones y por género, se observan diferencias importantes (Gráfica 2).

Las muertes evitables por vacuna y tratamiento preventivo son muy bajas, y la reducción obtenida en la década no deja de

GRAFICA 1. Frontera Norte: Estructura Porcentual de Defunciones Según Evitabilidad, 1980-1990

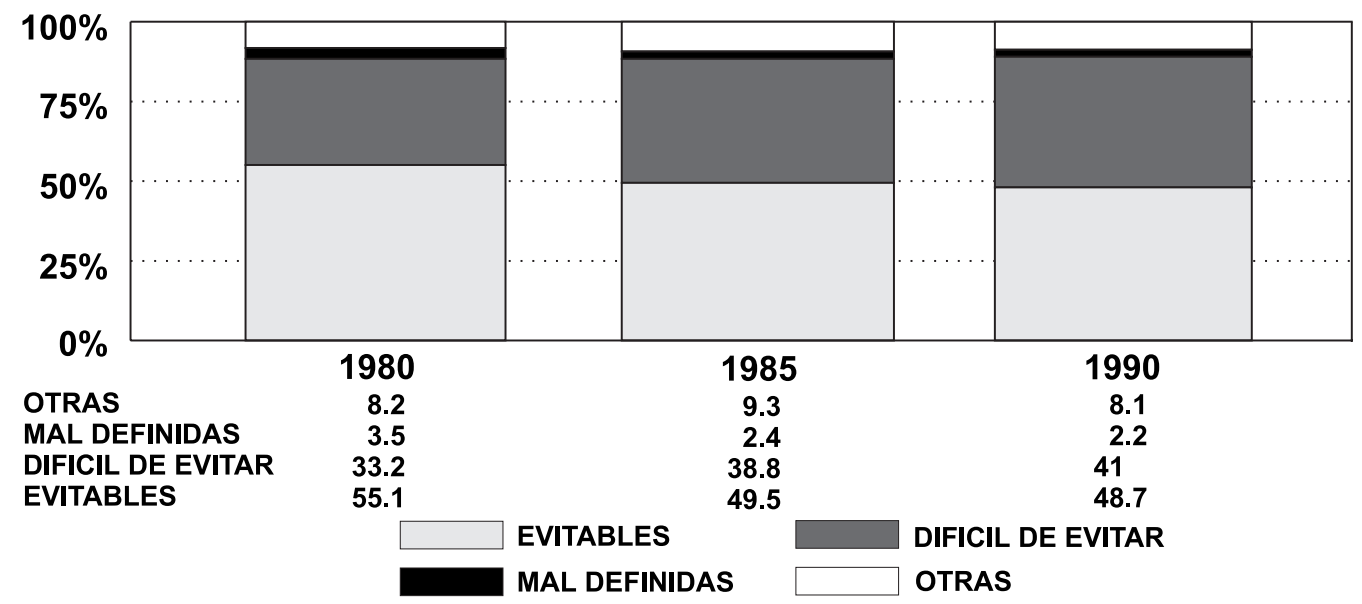

FUENTE: BASES DE MORTALIDAD INEGI-SSA 
GRAFICA 2. Frontera Norte: Estructura Porcentual de Defunciones Evitables Según Genero, Hombres y Mujeres, 1980-1990

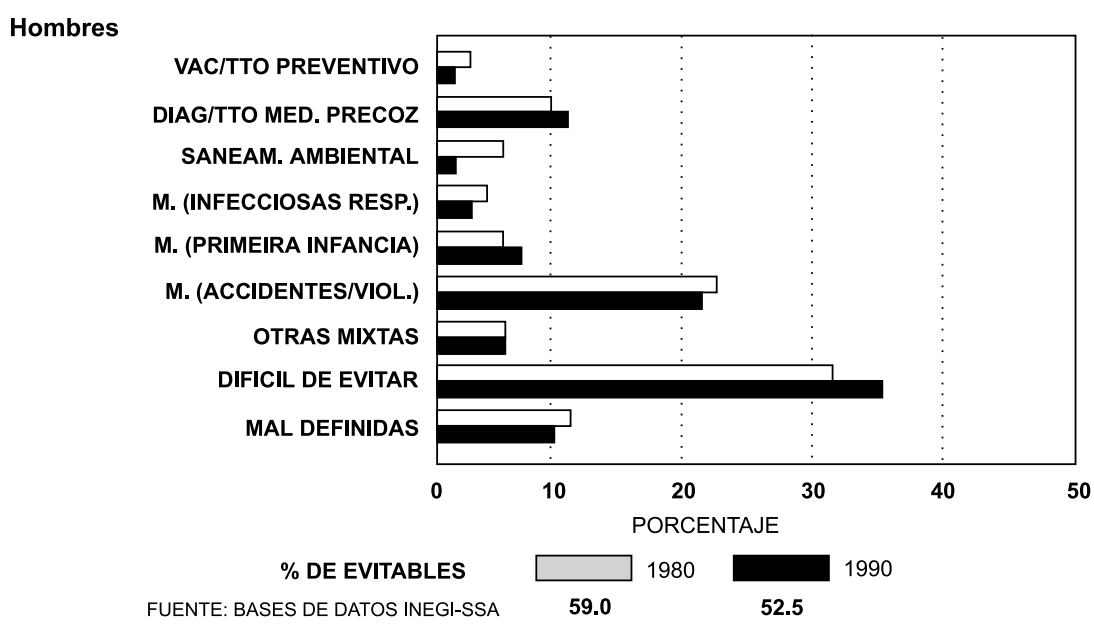

Mujeres

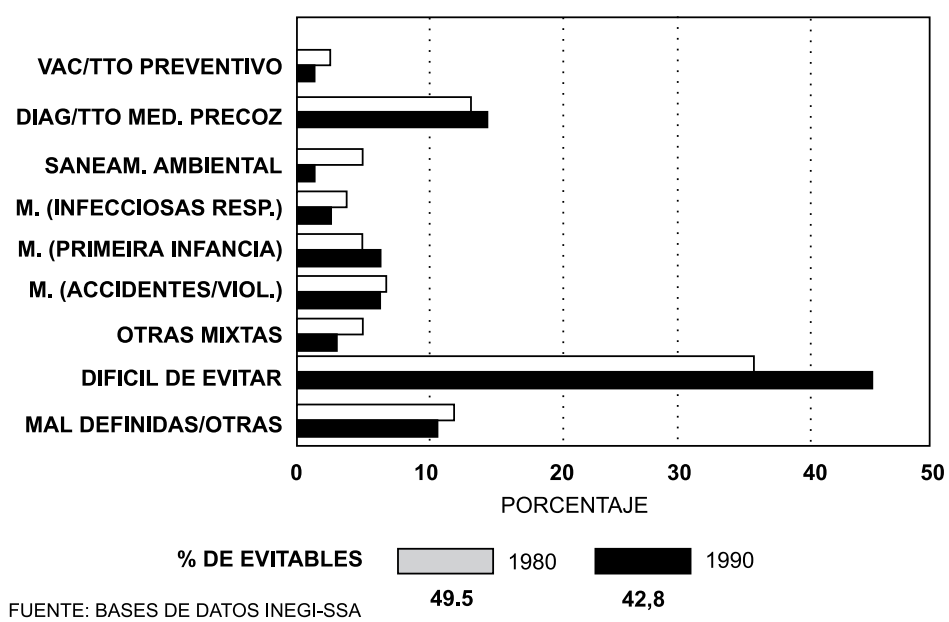

ser discreta. Sin embargo, han sido famosas las campañas nacionales de vacunación y se consideran relevantes las cifras sobre cuadros completos de inmunización. Las muertes que pudieron haber sido evitadas por diagnósticos médicos y tratamiento precoz superan en los hombres el $10 \%$ y en las mujeres el $15 \%$; en la década la tendencia fue al aumento. Si se considera que son enfermedades que tienen que ver con la prestación de servicios de salud y de qué tanto éstos responden a las necesidades y a la demanda de salud de la población, de las campañas de detección precoz del cáncer de mama y útero, próstata y de estómago, entonces es posible afirmar que los servicios de salud no han sido racionales en la manera de atender las necesidades de la población, sobre todo de las mujeres.

Las causas evitables con medidas de saneamiento ambiental han tenido un descenso de más de un $50 \%$ en la década. Sin embargo, todavía el $2.5 \%$ de las muertes se deben a factores de riesgo asociadas a precarias condiciones de vida y sin posibilidades de atención médica. Para 1990 Sonora presenta el mayor porcentaje; esto lo convierte en un estado prioritario en el control de factores de riesgo asociados al ambiente. 
Las causas de muerte evitables por un conjunto de medidas mixtas están subdivididas en cuatro subgrupos. Infecciosas y respiratorias que descendieron en un $50 \%$, siendo mayor su presencia en mujeres que en los hombres. En su conjunto, son las que menos aportan en 1990, pero que todavía representan cerca del $4 \%$ de las muertes totales. Las causas asociadas a la primera infancia han mantenido su aporte en el período y representan mas del 7\% de la mortalidad total. Baja California presenta el mayor porcentaje, si se trata de enfermedades fácilmente controlables, con buenos servicios de atención materno-infantil.

Como ya se había advertido anteriormente, son los accidentes y violencias las causas de muerte evitables que mayor porcentaje representan del total de muertes. Es el género masculino el que más muertes aporta $(21.4 \%$, en 1990), dos veces más que sus congéneres. La disminución en el período ha sido mínima y las diferencias entre estados no son despreciables y tienden a ahondarse. En Baja California, en 1980, una de cada cinco muertes se debió a causas definidas como accidentes o violencias; para 1990, dicha relación descendió, pero no lo hizo de manera importante. Mas preocupante es el caso de Chihuahua donde dichas causas se incrementaron en el período, pues su porcentaje pasó del 16 al 18\%, siendo, junto con Baja California, el estado que más aporta por este tipo de causas.

El cuarto subgrupo es residual y comprende enfermedades tales como la tuberculosis, las asociadas al embarazo, parto y puerperio y la cirrosis hepática. Su estabilidad en el tiempo y su aporte al total de muertes (más del 4\%) cuestionan seriamente la efectividad de la medicina preventiva en la región y de los programas de salud específicos, dirigidos a disminuir factores de riesgos de dichas enfermedades.

\section{Contribución a la Esperanza de Vida de las Causas Evitables}

Como ya se había advertido en la metodología, las estimaciones de la esperanza de vida al nacimiento que aquí se presentan serán utilizadas para mostrar diferencias. El nivel de la medición depende mucho del cumplimiento de los supuestos de las técnicas de evaluación y ajuste utilizadas. Es posible que las esperanzas de vida estén un poco por encima de su valor real (sobreestimadas).

La Gráfica 3 muestra un panorama general del comportamiento por género de la esperanza de vida en la década. Una ganancia promedio de 4.1 años en la esperanza de vida para una década es discreta, si se parte del hecho de que países de alta mortalidad y en procesos de disminución importantes logran un promedio de .5 años de ganancia anual.

La diferencia en la esperanza de vida al nacimiento entre géneros ha disminuido en .4 años. Aunque pequeña, es muy significativa esa disminución pues lo normal, lo que tradicionalmente se ha venido midiendo y observando, es una sobremortalidad en aumento, principalmente debido a una mayor mortalidad masculina por causas registradas como accidentes y violencias. Pero, como ya se dijo anteriormente, una mayor disminución a favor del género masculino ha hecho posible que la tendencia empiece a modificarse.

En efecto, mientras, en la década, la ganancia masculina en la esperanza de vida ascendió a 4.3 años, sus congéneres obtuvieron 3.95 años, lo que explica la disminución de la diferencia por género.

Estas ganancias fueron descompuestas según el aporte de cada grupo de causas evitables y grupos de edad. La Gráfica 4 es concluyente en el sentido de que todavía las ganancias en la sobrevivencia se vienen obteniendo por el descenso de la mortalidad infantil (más del 35\% de aporte a la ganancia en la esperanza de vida). Como es obvio, son las muertes infantiles evitadas las que mayor impacto tienen sobre la esperanza de vida, pero lo que está muy lejos de la obviedad es el hecho de que el componente principal de la disminución de la mortalidad dependa de los infantes, cuando la mortalidad adulta en México es todavía alta, si se le compara con otros países. La población de 50 años y mas ha logrado contribuir a dicha ganancia con más de un $20 \%$, siendo mayor el aporte femenino. 
Consecuente con la baja mortalidad juvenil, el aporte a la ganancia de lapoblación entre 1 y 9 años es muy baja. Aunque importante, se considera que el aporte a las ganancias, de la población entre 15 y 49 años, es insuficiente si realmente se buscan obtener perfiles de mortalidad que se correspondan con aquellos países y/o regiones que se caracterizan por una baja mortalidad.

GRAFICA 3. Frontera Norte: Esperanza de Vida al Nacimiento Según Genero, 1980-1990

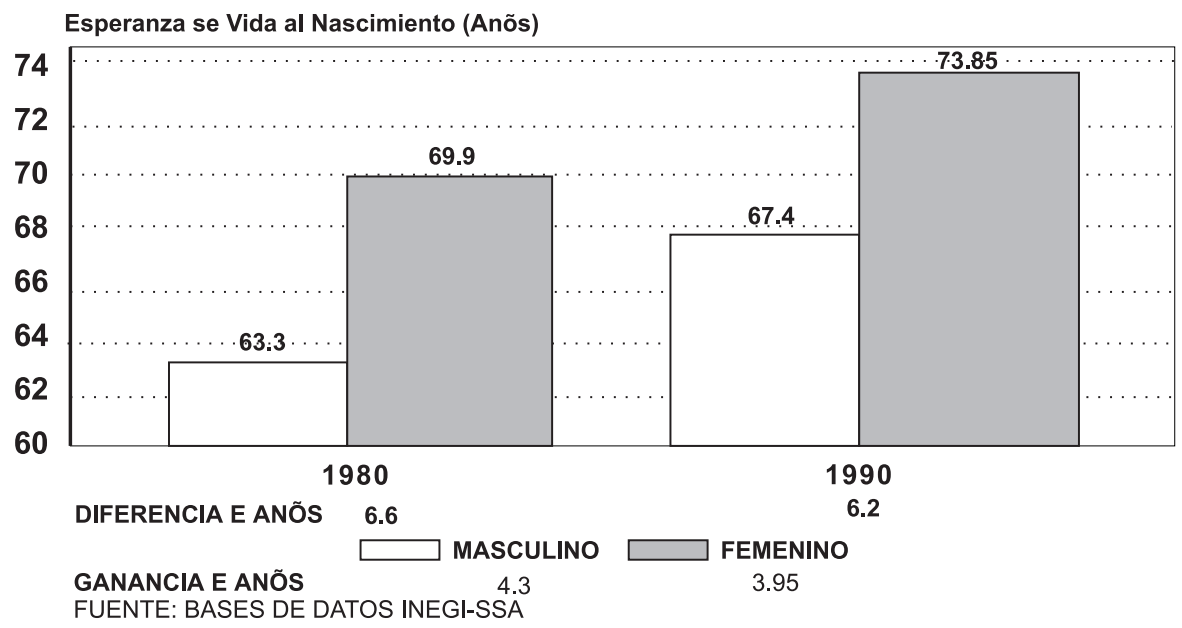

GRAFICA 4. Frontera Norte: Distribución Procentual de las Ganacias en la Esperanza de Vida Según enero y Edad

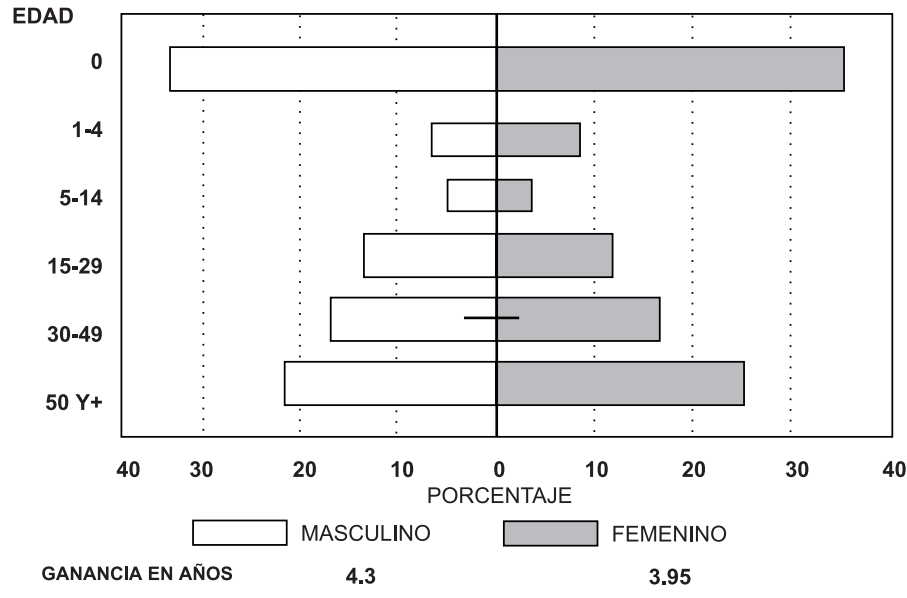

FUENTE: BASES DE DATOS INEGI-SSA

El mayor aporte a la ganancia en la esperanza de vida masculina se concentra en los grupos evitables: accidentes y violencias, saneamiento ambiental e infecciones respiratorias, en su orden. El aporte femenino lo hacen las de saneamiento ambiental, infecciosas respiratorias y diagnóstico y tratamiento médico precoz. Estas diferencias se pueden entender como un efecto directo de los distintos perfiles de mortalidad. 
Si se comparan estos resultados con la gráfica 2, que contiene las estructuras de la mortalidad por causas según evitabilidad, no solo se encuentra una asociación directa entre la evolución de la estructura y las ganancias en la esperanza de vida al nacimiento sino que también permite visualizar que, si se quiere reducir significativamente la mortalidad en el corto plazo, las acciones en salud tienenque estar dirigidas a incrementar el diagnóstico y tratamiento médico precoz (adaptación racional del sistema de salud) y dirigir la política económica y social a disminuir los factores que producen y reproducen la 'violencia social, responsable, enun alto porcentaje, de las muertes causadas por "accidentes, violencias y traumatismos".

En síntesis y como lo sugiere la gráfica 5, el perfil de mortalidad de la frontera norte dista mucho de acercarse a nuevos perfiles caracterizados por su asociación con los habitus de vida y provecho constante de las tecnologías médicas, más bien depende todavía de causas de muerte evitables con una mejor irradiación de los beneficios del desarrollo y con servicios de salud que efectivamente se correspondan con las necesidades de la población.

GRÁFICA 5. Frontera Norte: Distribución Porcentual de la Ganacia en la Esperanza de Vida Según Evitabilidad y Genero, 1980-1990

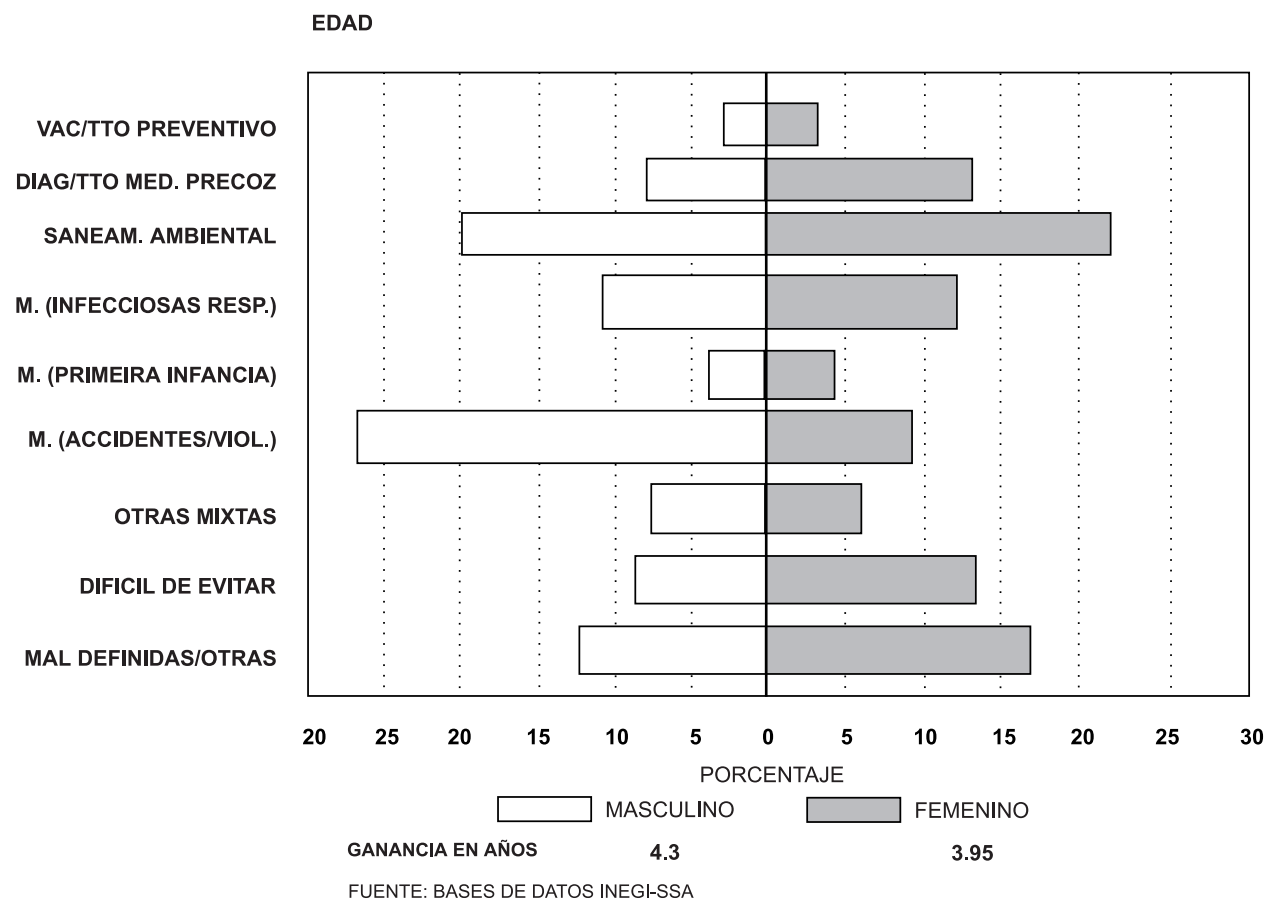

\section{DISCUSIONY CONCLUSIONES}

Si bien las diferencias encontradas se pueden asociar con las características socioeconómicas de los Estados y de las condiciones particulares que asume la frontera en su proceso de internacionalización, no se lo puede hacer de manera mecánica. No es posible atribuirle una relación de causa-efecto, aunque, sí tiene mucho que ver el nivel de desarrollo, hay que incluir variables 
políticas como la respuesta del sistema de salud a las demandas y necesidades de la población y la forma como la población concibe y opta por un marco de opciones en salud de acuerdo a sus particulares condiciones socio-económicas (García, 1992; Arredondo et al., 1992).

Es preocupante el alto porcentaje de accidentes y violencias encontrado en algunos estados o ciudades de la frontera y cuyo lugar de ocurrencia es la vía pública; normalmente se trata de fuerza de trabajo masculina. El hecho de que la fuerza de trabajo está muriendo en la calle no tiene justificación plausible desde las teorías de la modernización, mucho menos desde el punto de vista de la acumulación de capital. Esta situación debe ser analizada con más detalle por los estudiosos de la mortalidad al interior de cada entidad federativa y según lo distintos sectores de la economía.

La modernización no nos está matando de ataques al miocardio, sino de violencia que el mismo desarrollo produce y de enfermedades altamente diferenciables por género y ocupación. La población trabajadora muere dos veces más por homicidios, accidentes, envenenamientos y demás traumatismos que la población en general, y esto afecta mayormente al sexo masculino, ya que el $90 \%$ de las defunciones por este tipo de causas corresponden a este género. Lo que evidencia una forma desigual de contribuir al desarrollo y de apropiarse del bienestar social que este produce.

Teorías acerca de la "Transición epidemiológica" y de la "Transición demográfica" están todavía insuficientemente desarrolladas como para dar explicaciones a este tipo de fenómenos (Possas, 1993; Patarra, 1993).

$\mathrm{Si}$, en 13 años, no se logró disminuir de manera importante aquellas causas que realmente tienen posibilidades de disminuirse, es difícil pensar que en el corto plazo se lo pueda hacer. Aunque los procesos de salud-enfermedad-muerte son de larga duración y las tendencias deben ser analizadas en períodos lo mas largo posibles, creemos que esta serie de 13 años nos está mostrando al menos dos cosas importantes.
La primera es que algo pasa en el sistema de salud y en el acceso de la población a los beneficios del desarrollo que no hacen posible el disminuir las causas evitables. En segundo lugar, no es posible hablar de cambios de perfiles de mortalidad si tanto la estructura como el descenso de la mortalidad siguen dependiendo de causas que pudieron haber sido evitadas. Este criterio tiene más fuerza explicativa, aún desde el punto de vista de la vigilancia epidemiológica, que el de si las enfermedades son o no transmisibles, o las mal traducidas "comunicables".

Por otro lado, no se puede decir que se está en un régimen de baja mortalidad ya que las ganancias en la esperanza de vida por género, edad y condición de evitabilidad se corresponden con perfiles de alta mortalidad. En donde los descensos de mortalidad se consiguen en la población infantil y juvenil, situación observada a nivel nacional (Rodriguez, 1989), y todavía se está muy lejos de lograr bajas mortalidades.

Donde la mayor contribución a las ganancias en la esperanza de vida se logra en la población mayor de los 50 años, como está ocurriendo en algunos países europeos (Pollard, 1990). Cuando se compara la mortalidad de México con la de otros países de América Latina, éste se ubica en una situación intermedia (Arriaga, 1993).

Al contrario de la creencia popular, y como lo reporta Pollard para algunos países europeos (Pollard, 1990), la disminución relativa de los accidentes y violencias no sólo ha contribuido con importantes incrementos en la esperanza de vida sino que ha logrado disminuir, levemente, la brecha de la mortalidad entre géneros.

En todo caso, el hecho de que hoy la población masculina presente condiciones de mortalidad en que alrededor del $50 \%$ de las defunciones pudieron haber sido evitadas, y que sus congéneres apenas hayan pasado levemente esta barrera, debe ser considerado como un rasgo característico del perfil de la mortalidad en la Frontera Norte y es una realidad que debe preocupar al sector salud y a cualquier persona que pretenda mejorar las condiciones de salud de su población. 


\section{AGRADECIMIENTOS}

El Dr. Juan Francisco Molina Rodriguez participó activamente en la elaboración de la propuesta de agrupación de las causas evitables. Los estudiantes del Seminario de Población y Salud de la Maestría en Estudios de Población del Colegio de la Frontera Norte (COLEF) - Promoción 1993-1994 nos proporcionaron valiosa información cualitativa sobre la Frontera Norte y nos incentivaron con su entusiamo. Gracias a todos ellos.

\section{RESUMEN}

MOLINA, C. A. G. \& LOPEZ, M. V. L. Mortalidad Evitable. EI Caso de la Frontera Norte de México, 1980-1990. Cad. Saúde Públ., Rio de Janeiro, 11 (3): 395-407, jul/set, 1995.

Cuando se conciben las causas de muerte como el resultado de procesos mórbidos que pudieron haber sido evitados con distintos tipos de medidas económicas, sociales, de servicios de salud y los avances en el conocimiento científico y tecnológico, queda clara la existencia de una determinación estructural que impide que amplios sectores de la población transiten hacia "perfiles epidemiológicos modernos". Se usaron las bases de datos anuales sobre defunciones, entre 1979 y 1991, generadas por el INEGI/DGE-SSA para 5 estados fronterizos. Se ajustaron las defunciones según el procedimiento Preston-Coale y se construyeron tablas de vida para 1980/1990. Se encontraron los aportes de los grupos de causas evitables por sexo y edad a las ganancias en la esperanza de vida en el período según el procedimiento de Pollard. Entre los hallazgos se destacan: una discreta ganancia en la esperanza de vida, entre otras razones, debido a la permanencia de una alta contribución de defunciones que pudieron haber sido evitadas (50\%). En contra de lo esperado, el género masculino disminuyó la diferencia en la esperanza de vida con respecto a sus congéneres. La sobremortalidad masculina sigue siendo explicada por este tipo de causas, en donde los accidentes y violencias aportan altos porcentajes.

Palabras Clave: Mortalidad; Mortalidad por Causas; Evitabilidad

\section{REFERENCIAS BIBLIOGRAFICAS}

ARREDONDO, A.; CASTAÑEDA, X.; GARCIA, C.; ESCOBEDO, J. \& RAMIREZ, C., 1992. El enfoque de las Ciencias Sociales para el análisis de la morbi-mortalidad: Reflexiones para su enseñanza. Revista Mexicana de Educación Médica, 3: 16-29.

ARRIAGA, E., 1993. La Transición de la Mortalidad en Cuatro Países de América Latina. IV Conferencia Latinoamericana de Población. México, D.F.: ABEP/ CELADE/IUSSP/PROLAP/SOMEDE. (Mimeo.)

BENNETT, N. \& HORIUCHI, S.,1984. Estimación del grado de cabalidad en el registro de las muertes en una población cerrada. Revista Notas de Población, San José Costa Rica, 12: 11-33.

BRASS, W., 1974. Métodos para Estimar la Fecundidad y la Mortalidad en Poblaciones con Datos Limitados. Santiago de Chile: CELADE. (Serie E, 14)

CORONA, R., 1991. Principales características demográficas de la zona fronteriza del norte de México. Revista Frontera Norte, 3: 142-156.

CRUZ, R., 1992. La fuerza de trabajo en los mercados urbanos de la Frontera Norte. In: Cuadernos (Colegio de la Frontera Norte), vol 5, pp. 12-18, Tijuana, México: COLEF.

GARCIA, C., 1986. Mortalidad Infantil y Clases Sociales. El Caso de Medellín en la Década de los 70. Medellín, Colombia: Universidad Pontificia Bolivariana.

1992. La Desigualdad Social ante la Salud y la Muerte: El Caso de Medellín-Colombia. Una Visión Histórica. Tésis de Doctorado, México, D. F.: El Colegio de México.

GARCIA, C.; LOPEZ, M. V.; RODRIGUEZ, J., 1994. Frontera Norte: Perfiles de mortalidad en la década de los ochenta. Una interpretación sociodemográfiea. Revista SOCIOTAM, Tamaulipas, México, 4: 85-101.

GOMEZ, J. \& PARTIDA, V. 1993. Niveles de mortalidad infantil y fecundidad en México, por entidad federativa, 1990. Revista Mexicana de Sociología, LV: 97-135. 
JASPERS, D.; ORELLANA, H., 1991. Evaluación del uso de Estadisticas Vitales para el Estudio de la Mortalidad por Causas en América Latina. Seminar on Causes and Prevention of Adult Mortality in Developing Countries. Santiago de Chile: CELADE. (Mimeo.)

LOPEZ, M. V., 1991. Perfiles de Morbilidad en las Regiones de Antioquia, Colombia, Durante los Años Ochenta. Tesis de Maestría, México, D. F.: Universidad Autónoma Metropolitana (Xoch).

NEGRETE, J., 1990. La frontera morte de México en los informes presidenciales. Revista Frontera Norte, 2: 169-184.

OMS (Organización Mundial de la Salud), 1978. Clasifación Internacional de Enfermedades (CIE). Novena Revisión, Washington, D.C.: OMS.

ONU (Organización de las Naciones Unidas), 1986. Manual X. Técnicas Indirectas de Estimación Demográfica. Washington, D.C.: ONU.

ORTEGA, A., 1987. Tablas de Mortalidad. San José: CELADE.

PATARRA, N., 1993. Transição Demográfica: Novas Evidencias, Velhos Desafios. IV Conferencia Latinoamericana de Población. México, D.F.: ABEP/ CELADE/IUSSP/PROLAP/SOMEDE.

POLLARD, J., 1988. On the decomposition of changes in expectation of life and differentials in life expectancy. Demography, 25: 30-42.
1990. Cause of death and expectation of life: some international comparisons. In: Measurement and Analysis of Mortality. New Approaches (J. Vallin; S. D'souza \& A. Palloni, eds.), pp. 268-291, Oxford: Claredon Press.

POSSAS, C., 1993. A Dimensão Saúde da Transição Demográfica: Uma Discussão Conceitual. IV Conferencia Latinoamericana de Población. México, D.F.: ABEP/CELADE/IUSSP/PROLAPI SOMEDE.

PRESTON, S. \& COALE, A. 1984. Estructura por edades, crecimiento, salidas y entradas: una nueva sintesis. Revista Notas de Población, San José, Costa Rica, XII: 35-96.

RODRIGUEZ, L., 1989. México: Contribución de las Causas de Muerte al Cambio en la Esperanza de Vida, 1970-1982. Santiago de Chile: CELADE. (Serie B, 56)

TAUCHER, E., 1978. Mortalidad Infantil en Chile: Tendencias, Diferenciales y Causas. Santiago de Chile: CELADE.

1980. Chile: Mortalidad desde 1955 a 1975. Tendencias y causas. Santiago de Chile: CELADE. (Serie A, 162) 